\title{
A New Method Based on Undirected Graph and Polygon Triangulation to Prove the Four-color Theorem
}

\author{
Xue Geng, Liu Hui, Li Shixian, Chen Wei \\ Division of Information Resources \\ China Information Technology Security Evaluation Center \\ Beijing, China \\ $\{x g$, liuhui, lisx, chenw $\} @$ itsec.gov.cn
}

\begin{abstract}
The development of computer science and plane geometry brings a new favorable opportunity for the research about the Four-color Theorem. After converting original map to undirected graph, this paper proposes a new method based on undirected graph and polygon triangulation to prove the Four-color Theorem.
\end{abstract}

Keywords- the Four-color Theorem; undirected graph; polygon triangulation.

\section{INTRODUCTION}

The Four-color Theorem, which is also known as the Four-color Law, the Four-color Problem or the Four-color Conjecture ${ }^{[1]}$, is as famous as the Fermat Grand Theorem and the Goldbach Conjecture. Francis Guthrie was the exactly first people who put forward the Four-color Theorem in 1852 when he was engaged in coloring maps ${ }^{[2]}$. However, the formal expression about the Four-color Theorem can be described as below:

During coloring any map, we can ensure the countries having common boundary will be colored in different colors just using only four kinds of colors. It is noteworthy that the countries having only one or a finite number of common points can not be equated to the countries having common boundary ${ }^{[3]}$.For example, country A and country $\mathrm{C}$ in Figure 1 can not be considered as the countries having common boundary while country A and country B can.

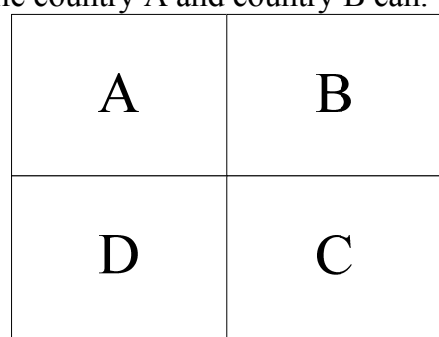

Figure 1. Example about countries having common boundary.

Generally speaking, we always consider the event that famous English mathematician Cayley proposed the Fourcolor Theorem to the London Mathematical Society in 1872 as the very beginning about the experts in area of Mathematics showing solicitude for this theorem ${ }^{[4]}$. And it has been taken more than one century for mathematicians to seek the way to prove it.
So far, there are three methods to prove this theorem: the first one is a computer assisted proof which is proposed by Appel and Haken in $1976^{[5]}$; the second method proposed by Seymour and Robertson in 1994 is an improved version of the first one and it can be checked artificially ${ }^{[6]}$; and the latest one proposed by Gonthier in 2005 is a formal proof with the whole logical steps ${ }^{[7]}$.

However, the first one is always considered as the official proof about the Four-color Theorem. So a lot of researchers still want to seek a new no-computer-assisted mathematical proof. This paper will propose a new method utilizing polygon triangulation after converting original map to undirected graph.

\section{CONVERSION}

In order to observe the relationships of the countries having common boundary, we convert the original map $\mathrm{G}_{0}$ (example shown as Figure 2) to undirected graph $\mathrm{G}$ (shown as Figure 3 ) at the very beginning.

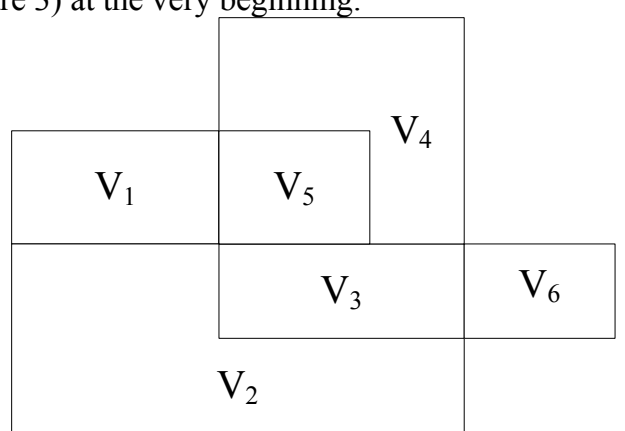

Figure 2. Example about the original map $\mathrm{G}_{0}$.

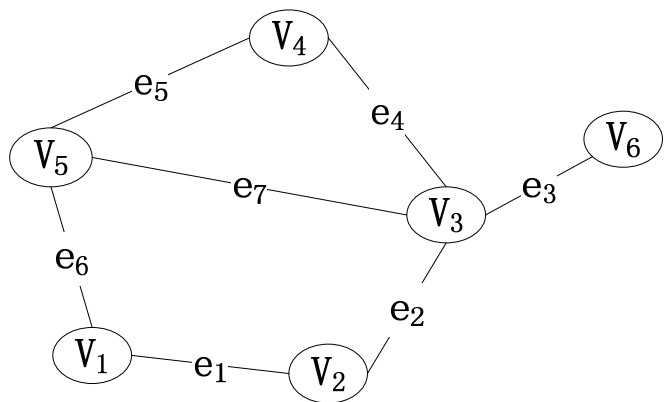

Figure 3. Example about undirected graph G. 
As shown in Figure 2 and Figure 3, considering the countries as the vertexes $\mathrm{v}_{\mathrm{i}}(\mathrm{i} \in \mathrm{N})$ while considering the common boundary as the edge $e_{i}(i \in N)$, the undirected graph $\mathrm{G}$ can be described as the following expression:

$$
\begin{aligned}
\mathrm{G} & =(\mathrm{V},\{\mathrm{E}\}) \\
\mathrm{V} & =\left\{\mathrm{v}_{1}, \mathrm{v}_{2}, \ldots\right\}, \quad \mathrm{E}=\left\{e_{1}, e_{2, \ldots\}}, \quad e_{\mathrm{i}=\left(\mathrm{v}_{\mathrm{m}}, \mathrm{v}_{\mathrm{n}}\right) \quad(\mathrm{i}, \mathrm{m},}\right.
\end{aligned}
$$
$\mathrm{n} \in \mathrm{N})$.

Then there will be two conclusions about the undirected graph G:

(1) According to the explanation about the common boundary, one common boundary will only belong to a couple of neighboring countries. So we can not find two intersecting edges in the undirected graph G. And this characteristic can be described as the following expression:

$$
e i \cap e j(i, j \in N)=\left\{\begin{array}{l}
v(e i=(v i, v), e j=(v, v j)) \\
\varphi(\text { any else })
\end{array}\right.
$$

(2) Because the neighboring relationships between the vertexes should be the most important aspect of the undirected graph $\mathrm{G}$, we totally can make sure that there are no straight angle in the undirected graph G. And this characteristic can be described as the following expression:

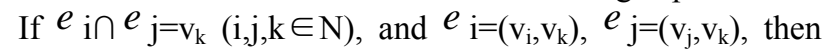
$\angle \mathrm{v}_{\mathrm{i}} \mathrm{v}_{\mathrm{k}} \mathrm{v}_{\mathrm{j}} \neq 180^{\circ}$

\section{ENCLOSED UNDIRECTED GRAPH}

This undirected graph $\mathrm{G}$ is an unclosed geometric figure. In order to utilize the triangulation characteristic of polygon, we must make some modifications by adding the fewest auxiliary edges for the nearby but unconnected vertexes to guarantee the undirected graph $\mathrm{G}$ become a closed geometric figure (example shown as Figure 4).

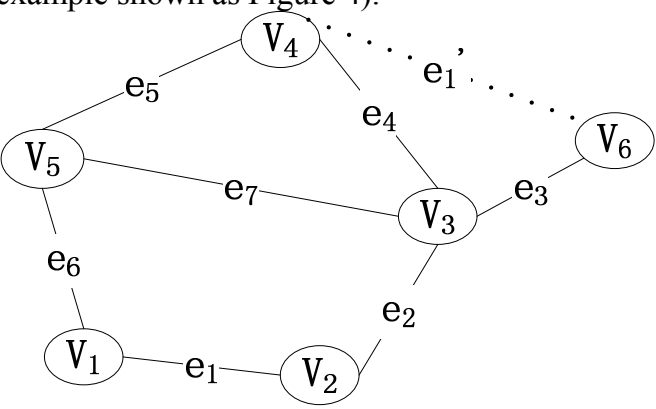

Figure 4. Example about new undirected graph G'.

And the new undirected graph G' should also satisfy the characteristic described as expression (2). And we can complete this process because of the conclusion described as expression (3).

Considering the set of adding auxiliary edges as E', the new undirected graph $G^{\prime}$ can be described as the following expression:

$$
\mathrm{G}^{\prime}=\mathrm{G}+\mathrm{E}^{\prime}=\left(\mathrm{V},\left\{\mathrm{E}+\mathrm{E}^{\prime}\right\}\right)
$$

Then there will be two conclusions about the new undirected graph $\mathrm{G}^{\prime}$ :
(1)The Degree of every vertex in the undirected graph G' is more than 2, and this characteristic can be described as the following expression:

$$
\mathrm{TD}\left(\mathrm{v}_{\mathrm{i}}\right) \geqslant 2
$$

(2) If we consider the vertexes of the undirected graph $\mathrm{G}$ ' as an intersection point of two edges, then we can consider each mesh of the undirected graph $\mathrm{G}^{\prime}$ as a polygon $\mathrm{S}_{\mathrm{i}}(\mathrm{i} \in \mathrm{N})$. So the undirected graph $G^{\prime}$ will be considered as a combinatorial figure described as the following expression:

$$
\begin{gathered}
\mathrm{G}^{\prime}=\mathrm{S}_{1} \cup \mathrm{S}_{2} \cup \mathrm{S}_{3} \cup \cdots \\
\mathrm{SV}_{1} \cup \mathrm{SV}_{2} \cup \mathrm{SV}_{3} \cup \cdots=\mathrm{V} \\
\mathrm{SE}_{1} \cup \mathrm{SE}_{2} \cup \mathrm{SE}_{3} \cup \cdots=\mathrm{E}+\mathrm{E}, \\
\mathrm{S}_{\mathrm{i}}=\left(\mathrm{SV}_{\mathrm{i}},\left\{\mathrm{SE}_{\mathrm{i}}\right\}\right) .
\end{gathered}
$$

\section{TRIANGULATION}

And next we need to make triangulation for each polygon mesh of the undirected graph G'. However, as the rectilinear polygon with least edges, triangle has an important inference described as following:

Triangle is the tiniest composition units of any enclosed rectilinear figure, in other words, a rectilinear polygon can separate into several disjoint triangles while all the vertexes of these triangles are the vertexes of original polygon.

It is the most common method to prove this inference that we first consider the enclosed rectilinear figure as the composition of a group of disjoint concave polygons and convex polygons, and then we separate every concave polygon into several disjoint convex polygons, and we separate every convex polygon into several disjoint triangles at last $^{[8]}$. However, there are also other methods such as based on determination of convex concave vertices ${ }^{[9]}$, based on BSP Tree ${ }^{[10]}$ and based on Monotonic Chain ${ }^{[11]}$.

According to this inference, we just can separate each polygon mesh of the undirected graph $G$ ' into a group of disjoint triangles. And the vertexes of all these triangles will be the vertexes of the undirected graph G'.

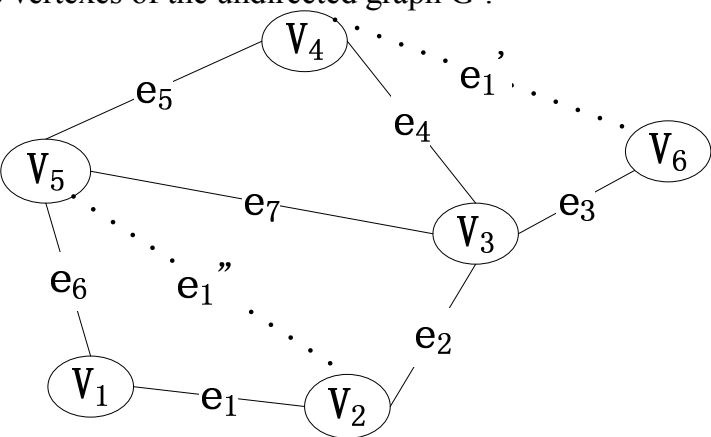

Figure 5. Example about new undirected graph G".

As shown in Figure 2, if we consider the set of adding auxiliary edges during triangulation as E", the new undirected graph G" can be described as the following expression:

$$
\mathrm{G}^{\prime \prime}=\mathrm{G}^{\prime}+\mathrm{E}^{\prime \prime}=\mathrm{G}+\mathrm{E}^{\prime}+\mathrm{E}^{\prime \prime}=\left(\mathrm{V},\left\{\mathrm{E}+\mathrm{E}^{\prime}+\mathrm{E}^{\prime \prime}\right\}\right)
$$

Furthermore, if we consider each triangle mesh of the undirected graph $\mathrm{G}$ " as $\operatorname{Ti}(\mathrm{i} \in \mathrm{N})$, the new undirected graph G" can be described as the following expression: 


$$
\begin{gathered}
\mathrm{G}^{\prime \prime}=\mathrm{T}_{1} \cup \mathrm{T}_{2} \cup \mathrm{T}_{3} \cup \cdots \\
\mathrm{TV}_{1} \cup \mathrm{TV}_{2} \cup \mathrm{TV}_{3} \cup \cdots=\mathrm{V} \\
\mathrm{TE}_{1} \cup \mathrm{TE}_{2} \cup \mathrm{TE}_{3} \cup \cdots=\mathrm{E}+\mathrm{E}^{\prime}+\mathrm{E}^{\prime}, \\
\mathrm{T}_{\mathrm{i}}=\left(\mathrm{TV}_{\mathrm{i}},\left\{\mathrm{TE}_{\mathrm{i}}\right\}\right) .
\end{gathered}
$$

\section{COLORATION}

After the conversion from original undirected graph $\mathrm{G}$ to the new undirected graph G", we will adopt "Triangular Element" method, which means that we use three different colors to color the three vertexes of a triangle mesh while the color of third vertex is determined by the other two ${ }^{[12]}$, to color all the vertexes of undirected graph G” just beginning with two vertexes of a random triangle mesh.

According to the expression (2), it must be noticed that any vertex of the new undirected graph G" can be the common vertex of several triangle meshes, but any edge of the new undirected graph G" just can be the common edge of two neighbor triangle meshes at most. That is to say, the three vertexes of any triangle mesh can connect to at most one other vertex at the same time, and they can not connect with more than two other vertexes at the same time.

However, the only special case during the coloration will be described as Figure 6: $2 n+1$ edges $(n \in N)$ have one common vertex while these $2 n+1$ edges are the common edges of $2 n+1$ neighbor triangle meshes, then there will be one vertex must be colored with a fourth color.

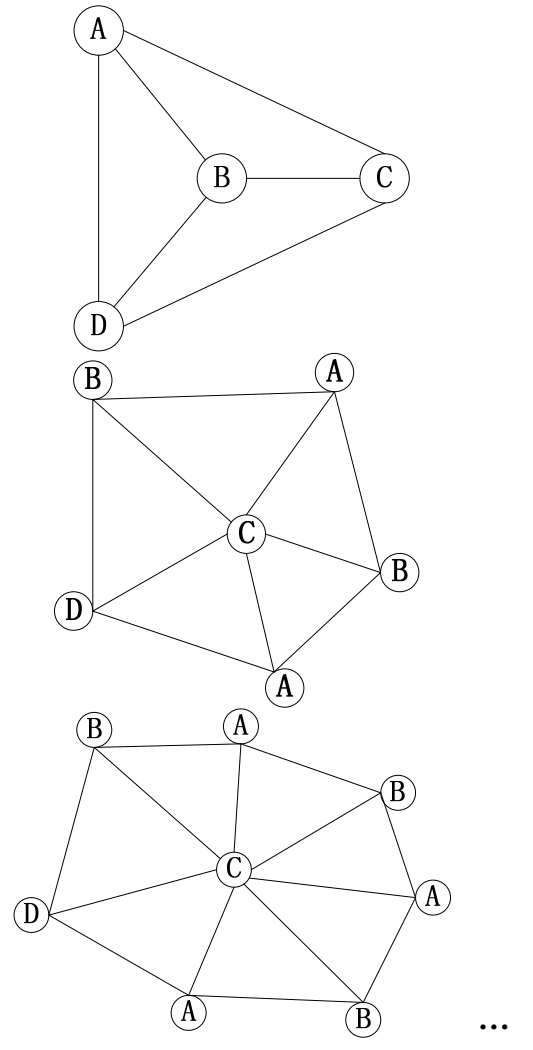

Figure 6. Examples about the special case.
Every time the special case has come up, after using the fourth color just once, we continue the coloration for the rest vertexes of the new undirected graph G" by utilizing the original three colors until we finish the whole coloration.

However, we realize the coloration for all the vertexes of the new undirected graph G" just using no more than four kinds of colors. According to the expression (9), there are more edges in the new undirected graph $G$ " than in the original undirected graph $\mathrm{G}$ while both the new undirected graph G" and the original undirected graph $G$ have the same number of vertexes.

So if we remove the edges of set E' and E" in the new undirected graph G", we will get the original undirected graph $\mathrm{G}$, and we just reduce the relationship between the vertexes, but there is no effect on the coloration. That means we realize the coloration for all the countries of the original map $\mathrm{G}_{0}$ just using no more than four colors. However, the case show as Figure 6 proves that the least number of colors is four.

\section{CONCLUSION}

This paper proposes a new method based on undirected graph and polygon triangulation, and we just utilize the basic characteristic of triangle to prove the Four-color Theorem. Actually, with the corresponding 2D undirected graph, this method is suitable for not only $2 \mathrm{D}$ but also $3 \mathrm{D}$ maps.

\section{REFERENCES}

[1] Ouyang Guangzhong. The Four-color Problem[M]. People Education Press. 1981.

[2] K. May. The Origin of the Four- Color Conjecture[J]. Isis.1965, 56, pp346-348.

[3] Kempe A B. On the geographical problem of the four colors[J]. Amer J Math, 1879(2):193-200.

[4] A. Cayley. On the Colouring of Maps[J] . Proceedings of the Royal Geographical Society ( New Series) . 1879( 1) : 259- 261.

[5] Appel K I, HakenW. Every planar map is four colourable [J ]. Bull. Am. M ath. Soc. 1976, 82: 711-712.

[6] N. Robertson, D. Sanders, P. Seymour, R. Thomas. The Four- Color Theorem[J]. Journal of Combinatorial Theory (Ser. B) . 1997,70: 244.

[7] G. Gonthier. Formal Proof: The Four- Color Theorem[J]. Notices of the AMS. 2008, 55( 11) : 1382- 1393.

[8] T. Pavlidis. Algorithms for graphics and image processing[J]. Rockville, Computer Science, 1982.

[9] Ma Xiaohu, Pan Zhigeng. Triangulation of simple polygon based on determination of convex concave vertices[J]. Journal of ComputerAided Design \& Computer Graphics, 1999,11(1) :1-3.

[10] Liu Qiang, Li Deren. A Recursive Algorithm for Triangulation of Arbitrary Polygons Based on BSP Tree[J]. Journal of Wuhan University (Information Science Edition),2002,27(5) :528-532.

[11] Zhang Yuping, Jaing Shouwei. Efficient Approach of Convex Hull Triangulation Based on Monotonic Chain[J]. Journal of Nanjing University of Science and Technology, 2004,28 (6) : 590-594.

[12] Xue Geng, Fan Xiumei, Liao Lejian. The Application of Mapcoloring Technology to Backbone Routing System[C]. Proceedings of the 2009 International Symposium on Computer Network and Multimedia Technology. CNMT 2009, p 1136-9, 2009 